\title{
Modeling Quantum Behavior in the Framework of Permutation Groups
}

\author{
Vladimir Kornyak $^{1, \star}$ \\ ${ }^{1}$ Laboratory of Information Technologies, Joint Institute for Nuclear Research, \\ 141980 Dubna, Moscow Region, Russia
}

\begin{abstract}
Quantum-mechanical concepts can be formulated in constructive finite terms without loss of their empirical content if we replace a general unitary group by a unitary representation of a finite group. Any linear representation of a finite group can be realized as a subrepresentation of a permutation representation. Thus, quantum-mechanical problems can be expressed in terms of permutation groups. This approach allows us to clarify the meaning of a number of physical concepts. Combining methods of computational group theory with Monte Carlo simulation we study a model based on representations of permutation groups.
\end{abstract}

\section{Introduction}

Since the time of Newton, differential calculus demonstrates high efficiency in describing physical phenomena. However, the infinitesimal analysis introduces infinities in the physical theories. This is often considered as a serious conceptual flaw: recall, for example, Dirac's frequently quoted claim that the most important challenge in physics is "to get rid of infinity". Moreover, differential calculus, being, in fact, a kind of approximation, may lead to descriptive losses in some problems - an illustrative example is given below in Sec. 3.1. In the paper, we describe a constructive version of the quantum formalism that does not involve any concepts associated with actual infinities.

The main part of the paper starts with Sec. 2, which contains a summary of the basic concepts of the standard quantum mechanics with emphasis on the aspects important for our purposes.

Sec. 3 describes a constructive modification of the quantum formalism. We start with replacing a continuous group of symmetries of quantum states by a finite group. The natural consequence of this replacement is unitarity, since any linear representation of a finite group is unitary. Further, any finite group is naturally associated with some cyclotomic field. Generally, a cyclotomic field is a dense subfield of the field of complex numbers. This can be regarded as an explanation of the presence of complex numbers in the quantum formalism. Any linear representation of a finite group over the associated cyclotomic field can be obtained from a permutation action of the group on vectors with natural components by projecting into a suitable invariant subspace. All this allows us to reproduce all the elements of the quantum formalism in invariant subspaces of the permutation representations.

In Sec. 4 we consider a model of quantum evolution inspired by the quantum Zeno effect - the most convincing manifestation of the role of observation in the dynamics of quantum systems. The model represents the quantum evolution as a sequence of observations with unitary transitions between them. The standard quantum mechanics assumes a single deterministic unitary transition between observations. In our model we generalize this assumption. We treat a unitary transition as a kind

\footnotetext{
^e-mail: vkornyak@gmail.com
} 
of gauge connection - a way of identifying indistinguishable entities at different times. A priori, any unitary transformation can be used as a data identification rule. So, we assume that all unitary transformations participate in transitions between observations with appropriate weights. We call a unitary evolution dominant if it provides the maximum transition probability. ${ }^{1}$ The Monte Carlo simulation shows a sharp dominance of such evolutions over other evolutions. To compare with a continuous description, we present also the Lagrangian of the continuum approximation of the model.

\section{Formalism of quantum mechanics}

Here is a brief outline of the basic concepts of the quantum mechanics. We divide these concepts into three categories: states, observations and measurements, and time evolution.

\subsection{States}

A pure quantum state is a ray in a Hilbert space $\mathcal{H}$ over the complex field $\mathbb{C}$, i.e., an equivalence class of vectors $|\psi\rangle \in \mathcal{H}$ with respect to the equivalence relation $|\psi\rangle \sim a|\psi\rangle$, where $a \in \mathbb{C}, a \neq 0$. We can reduce the equivalence classes by normalization: $|\psi\rangle \sim \mathrm{e}^{\mathrm{i} \alpha}|\psi\rangle,\|\psi\|=1, \alpha \in \mathbb{R}$. Finally, we can eliminate the phase "degree of freedom" $\alpha$ by transition to the rank one projector $\Pi_{\psi}=|\psi\rangle\langle\psi|$, which is a special case of a density matrix.

A mixed quantum state is described by a general density matrix $\rho$ characterized by three properties: (a) $\rho=\rho^{\dagger}$, (b) $\langle\psi|\rho| \psi\rangle \geq 0$ for any $|\psi\rangle \in \mathcal{H}$, (c) $\operatorname{tr} \rho=1$. In fact, any mixed state is a weighted mixture of pure states, i.e., its density matrix can be represented as a weighted sum of rank one projectors. We will denote the set of all density matrices by $\mathcal{D}(\mathcal{H})$.

The Hilbert space of a composite system, $X Y=X \times Y$, is the tensor product of the Hilbert spaces for its constituents: $\mathcal{H}_{X Y}=\mathcal{H}_{X} \otimes \mathcal{H}_{Y}$. The states of a composite system, $\mathcal{D}\left(\mathcal{H}_{X Y}\right)$, are classified into two types: separable and entangled states. The set of separable states, $\mathcal{D}_{\mathrm{S}}\left(\mathcal{H}_{X Y}\right)$, consists of the states $\rho_{X Y} \in \mathcal{D}\left(\mathcal{H}_{X Y}\right)$ that can be represented as weighted sums of the tensor products of states of the constituents: $\rho_{X Y}=\sum_{k} w_{k} \rho_{X}^{k} \otimes \rho_{Y}^{k}, \quad w_{k} \geq 0, \quad \sum_{k} w_{k}=1$. The set of entangled states, $\mathcal{D}_{\mathrm{E}}\left(\mathcal{H}_{X Y}\right)$, is by definition the complement of $\mathcal{D}_{\mathrm{S}}\left(\mathcal{H}_{X Y}\right)$ in the set of all states: $\mathcal{D}_{\mathrm{E}}\left(\mathcal{H}_{X Y}\right)=\mathcal{D}\left(\mathcal{H}_{X Y}\right) \backslash \mathcal{D}_{\mathrm{S}}\left(\mathcal{H}_{X Y}\right)$.

\subsection{Observations and measurements}

The terms 'observation' and 'measurement' are often used as synonyms. However, it makes sense to separate these concepts: we treat observation as a more general concept which does not imply, in contrast to measurement, obtaining numerical information.

An observation is the detection ("click of detector") of a system found in the state $\rho$ in the subspace $\mathcal{S} \leq \mathcal{H}$. The mathematical abstraction of the "detector in the subspace" $\mathcal{S}$ of a Hilbert space is the operator of projection, $\Pi_{\mathcal{S}}$, into this subspace. The result of quantum observation is random and its statistics is described by a probability measure defined on subspaces of the Hilbert space. Any such measure $\mu(\cdot)$ must be additive on any set of mutually orthogonal subspaces of a Hilbert space: if, e.g., $\mathcal{A}$ and $\mathcal{B}$ are mutually orthogonal subspaces, then $\mu(\operatorname{span}(\mathcal{A}, \mathcal{B}))=\mu(\mathcal{A})+\mu(\mathcal{B})$. Gleason proved [1] that, except the case $\operatorname{dim} \mathcal{H}=2$, the only such measures have the form $\mu_{\rho}(\mathcal{S})=\operatorname{tr}\left(\rho \Pi_{\mathcal{S}}\right)$, where $\rho$ is an arbitrary density matrix. If, in particular, $\rho$ describes a pure state, $\rho=|\psi\rangle\langle\psi|$, and $\mathcal{S}$ is one-dimensional, $\mathcal{S}=\operatorname{span}(|\varphi\rangle)$, we come to the familiar Born rule: $\operatorname{tr}\left(\rho \Pi_{\mathcal{S}}\right)=\mathbf{P}_{\text {Born }}=|\langle\varphi \mid \psi\rangle|^{2}$.

A measurement is a special case of observation, when the partition of a Hilbert space into mutually orthogonal subspaces is provided by a Hermitian operator $A$. Any such operator can be written as $A=\sum_{k} a_{k} \Pi_{e_{k}}$, where $a_{1}, a_{2}, \ldots \in \mathbb{R}$ is the spectrum of $A$, and $e_{1}, e_{2}, \ldots$ is an orthonormal basis of eigenvectors of $A$. "Click of the detector" $\Pi_{e_{k}}$ is interpreted as that the eigenvalue $a_{k}$ is the result of the measurement. The mean for multiple measurements tends to the expectation value of $A$ in the state $\rho:\langle A\rangle_{\rho}=\operatorname{tr}(\rho A)$.

\footnotetext{
${ }^{1}$ In fact, the principle of least action in physical theories implies the selection of dominant evolutions among all possible ("virtual") evolutions. The apparent determinism of these evolutions can be explained by the sharpness of their dominance.
} 


\subsection{Time evolution}

The time evolution of a quantum system is a unitary transformation of data between observations. For a density matrix, the unitary evolution takes the form

$$
\rho_{t^{\prime}}=U_{t^{\prime} t} \rho_{t} U_{t^{\prime} t}^{\dagger},
$$

where $\rho_{t}$ is the state after observation at the time $t, \rho_{t^{\prime}}$ is the state before observation at the time $t^{\prime}$, and $U_{t^{\prime} t}$ is the unitary transition between the observation times $t$ and $t^{\prime}$. In the standard quantum formalism, the time is considered as a continuous parameter, and the relation (1) becomes the von Neumann equation in the infinitesimal limit. The evolution of a pure state can be written as $\left|\psi_{t^{\prime}}\right\rangle=$ $U_{t^{\prime} t}\left|\psi_{t}\right\rangle$, and the corresponding infinitesimal limit is the Schrödinger equation. To emphasize the role of the observation in the quantum physics, we note that the unitary evolution is simply a change of coordinates in the Hilbert space and it is not sufficient to describe observable physical phenomena.

\subsection{Emergence of geometry within large Hilbert space via entanglement}

The quantum-mechanical theory does not need the geometric space as a fundamental concept - everything can be formulated using only the Hilbert space formalism. In this view, the observed geometry must emerge as an approximation. The currently popular idea [2-4] of the emergence of geometry within a Hilbert space is based on the notion of entanglement. Briefly, the scheme of extracting geometric manifold from the entanglement structure of a quantum state $\rho$ in a Hilbert space $\mathcal{H}$ is as follows:

- The Hilbert space decomposes into a large number of tensor factors: $\mathcal{H}=\otimes_{x} \mathcal{H}_{x}, x \in X$. Each factor is treated as a point (or bulk) of geometric space to be built. A graph $G$ - called tensor network - with vertices $x \in X$ and edges $\{x, y\} \in X \times X$ is introduced.

- The edges of $G$ are assigned weights based on a measure of entanglement, a function that vanishes on separable states and is positive on entangled states. A typical such measure is the mutual information: $I\left(\rho_{x y}\right)=S\left(\rho_{x}\right)+S\left(\rho_{y}\right)-S\left(\rho_{x y}\right)$, where $\rho_{x}$ denotes the result of taking traces of $\rho$ over all tensor factors except for the $x$-th (and similarly for $\left.\rho_{y}, \rho_{x y}\right) ; \quad S(a)=-\operatorname{tr}(a \log a)$ is the von Neumann entropy. The graph $G$ is supplied with a metric derived from the weights of the edges.

- Finally, the graph $G$ is approximately isometrically embedded in a smooth metric manifold of an as small as possible dimension using algorithms like the multidimensional scaling (MDS).

\section{Constructive modification of the quantum formalism}

David Hilbert, a prominent advocate of the free use of the concept of infinity in mathematics, wrote the following about the relation of the infinite to the reality: "Our principal result is that the infinite is nowhere to be found in reality. It neither exists in nature nor provides a legitimate basis for rational thought - a remarkable harmony between being and thought." Adopting this view, we reformulate the quantum formalism in constructive finite terms without distorting its empirical content [5-7].

\subsection{Losses due to continuum and differential calculus}

Differential calculus (including differential equations, differential geometry, etc.) forms the basis of the mathematical methods in physics. The applicability of the differential calculus is based on the assumption that any relevant function can be approximated by linear relations at small scales. This assumption simplifies many problems in physics and mathematics, but at the cost of loss of completeness.

As an example, consider the problem of classifying simple groups. The concept of group is an abstraction of the properties of permutations (also called one-to-one mappings or bijections) of a set. Namely, an abstract group is a set with an associative operation, an identity element, and an 
invertibility for each element. There are two most common additional assumptions that make the notion of a group more meaningful: (a) the group is a differentiable manifold - such a group is called Lie group; (b) the group is finite. The empirical physics is insensitive to the assumption (b) ultimately, any empirical description is reduced to a finite set of data. On the contrary, the assumption (a) implies severe constraints on the possible physical models.

The problem of the classification of the simple groups ${ }^{2}$ under the assumption (a) turned out to be rather easy and was solved by two people (Killing and Cartan) in a few years. The result is four infinite series: $A_{n}, B_{n}, C_{n}, D_{n}$; and five exceptional groups: $E_{6}, E_{7}, E_{8}, F_{4}, G_{2}$.

The solution of the classification problem under the assumption (b) required the efforts of about a hundred people for over a hundred years [8]. But the result - "the enormous theorem" - turned out to be much richer. The list of finite simple groups contains $16+1+1$ infinite series:

- groups of Lie type: $A_{n}(q), B_{n}(q), C_{n}(q), D_{n}(q), E_{6}(q), E_{7}(q), E_{8}(q), F_{4}(q), G_{2}(q)$, ${ }^{2} A_{n}\left(q^{2}\right),{ }^{2} B_{n}\left(2^{2 n+1}\right),{ }^{2} D_{n}\left(q^{2}\right),{ }^{3} D_{4}\left(q^{3}\right),{ }^{2} E_{6}\left(q^{2}\right),{ }^{2} F_{4}\left(2^{2 n+1}\right),{ }^{2} G_{2}\left(3^{2 n+1}\right) ;$

- cyclic groups of prime order, $\mathbb{Z}_{p}$;

- alternating groups, $A_{n}, n \geq 5$;

and 26 sporadic groups: $M_{11}, M_{12}, M_{22}, M_{23}, M_{24}, J_{1}, J_{2}, J_{3}, J_{4}, C o_{1}, C o_{2}, C o_{3}$, $\mathrm{Fi}_{22}, \mathrm{Fi}_{23}, \mathrm{Fi}_{24}, \mathrm{HS}, \mathrm{McL}, \mathrm{He}, \mathrm{Ru}, \mathrm{Suz}, \mathrm{O}^{\prime} \mathrm{N}, \mathrm{HN}, \mathrm{Ly}, \mathrm{Th}, \mathrm{B}, \mathrm{M}$.

Note that finite groups have an advantage over the Lie groups in the sense that in the empirical applications any Lie group can be modeled by some finite group, but not vice versa.

\subsection{Replacing a unitary group by a finite group}

The main non-constructive element of the standard quantum formalism is the unitary group $\mathrm{U}(n)$, a set of cardinality of the continuum.

Formally, the group $\mathrm{U}(n)$ can be replaced by some finite group which is empirically equivalent to $\mathrm{U}(n)$ as follows. From the theory of quantum computing it is known that $\mathrm{U}(n)$ contains a dense finitely generated - and, hence, countable - matrix subgroup $\bigcup_{*}(n)$. The group $\bigcup_{*}(n)$ is residually finite, i.e., it has a reach set of non-trivial homomorphisms to finite groups.

In essence, it is more natural to assume that, at the fundamental level, there are finite symmetry groups and that the $\mathrm{U}(n)$ 's are just continuum approximations of their unitary representations.

The following properties of the finite groups are important for our purposes:

- any finite group is a subgroup of a symmetric group,

- any linear representation of a finite group is unitary,

- any linear representation is a subrepresentation of some permutation representation.

\section{3 "Physical" numbers}

The basic number system in quantum formalism is the complex field $\mathbb{C}$. This non-constructive field can be obtained as a metric completion of many algebraic extensions of the rational numbers. We consider here constructive numbers that are closely related to the finite groups and are based on two primitives with clear intuitive meanings:

1. natural numbers ("counters"): $\mathbb{N}=\{0,1, \ldots\}$;

2. kth roots of unity ("algebraic form of the idea of $k$-periodicity"): $r_{k} \mid r_{k}^{k}=1$.

These basic concepts are sufficient to represent all physically meaningful numbers.

\footnotetext{
${ }^{2}$ The simple groups, i.e., groups that do not contain nontrivial normal subgroups, are "building blocks" for all other groups.

${ }^{3}$ There are $k$ different $k$ th roots of unity. A $k$ th root of unity is called primitive if $r_{k}^{m} \neq 1$ for any $m, 0<m<k$.
} 
We start by introducing $\mathbb{N}\left[r_{k}\right]$, the extension of the semiring $\mathbb{N}$ by primitive $k$ th root of unity. $\mathbb{N}\left[\mathrm{r}_{k}\right]$ is a ring if $k \geq 2$. This construction allows, in particular, to add negative numbers to the naturals: $\mathbb{Z}=\mathbb{N}\left[r_{2}\right]$ is the extension of $\mathbb{N}$ by the primitive square root of unity. Further, by a standard mathematical procedure, we obtain the kth cyclotomic field $\mathbb{Q}\left(r_{k}\right)$ as the fraction field of the ring $\mathbb{N}\left[r_{k}\right]$. If $k \geq 3$, then the field $\mathbb{Q}\left(r_{k}\right)$ is a dense subfield of $\mathbb{C}$, i.e., (constructive) cyclotomic fields are empirically indistinguishable from the (non-constructive) complex field. Note that $\mathbb{Q} \cong \mathbb{Q}\left(r_{2}\right)$.

The importance of the cyclotomic numbers for the constructive quantum mechanics is explained by the following. Let us recall some terms [9]. The exponent of a group $\mathrm{G}$ is the least common multiple of the orders of its elements. A splitting field for a group $\mathrm{G}$ is a field that allows to split completely any linear representation of $\mathrm{G}$ into irreducible components. A minimal splitting field is a splitting field that does not contain proper splitting subfields. Although a minimal splitting field for a given group $\mathrm{G}$ may be non-unique, any minimal splitting field is a subfield of some cyclotomic field $\mathbb{Q}\left(r_{k}\right)$, where $k$ is a divisor of the exponent of $G$. Thus, to work with any unitary representation of $G$ it is sufficient to use the $k$ th cyclotomic field, where $k$ is related to the structure of $\mathrm{G}$.

\subsection{Constructive representations of a finite group}

Let a group $\mathrm{G}$ act by permutations on a set $\Omega,|\Omega|=\mathrm{N}$. If we assume that the elements of $\Omega$ are "types" of some discrete entities ("ontological entities", "elements of reality"), then the collections of these entities can be described as elements of the module $H=\mathbb{N}^{\mathrm{N}}$ over the semiring $\mathbb{N}$ with the basis $\Omega$. The decomposition of the action of $\mathrm{G}$ on the module $H$ into irreducible components reflects the structure of the invariants of the action. In order for the decomposition to be complete, it is necessary to extend the semiring $\mathbb{N}$ to a splitting field, e.g., to a cyclotomic field $\mathbb{Q}\left(r_{k}\right)$, where $k$ is a suitable divisor of the exponent of $\mathrm{G}$. With such an extension of the scalars, the module $H$ is transformed into the Hilbert space $\mathcal{H}$ over $\mathbb{Q}\left(r_{k}\right)$. This construction, with a suitable choice of the permutation domain $\Omega$, allows us to obtain any representation of the group $\mathrm{G}$ in some invariant subspace of the Hilbert space $\mathcal{H}$. We obtain "quantum mechanics" within an invariant subspace if, in addition to unitary evolutions, projective measurements are also restricted to this subspace.

The above is illustrated in Figure 1 by the example of the natural action of the symmetric group $\mathrm{S}_{\mathrm{N}}$ on the set $\Omega=\left\{e_{1}, \ldots, e_{\mathrm{N}}\right\}$. Note, that any symmetric group is a rational-representation group, i.e., the field of rational numbers $\mathbb{Q}$ is a splitting field for $S_{N}$.

\section{Modeling quantum evolution}

The fundamental discrete time $\mathcal{T}$ is represented by an ordered sequence of integers: $\mathcal{T}=\mathbb{N}$ or $\mathcal{T}=\mathbb{Z}$. We define a finite sequence of "instants of observations" as a subsequence of $\mathcal{T}$ :

$$
\left[t_{0}, t_{1}, \ldots, t_{k-1}, t_{k}, \ldots, t_{n}\right] .
$$

The data of the model of the quantum evolution includes the sequence of the length $n+1$ for the states

$$
\left[\rho_{0}, \rho_{1}, \ldots, \rho_{k-1}, \rho_{k}, \ldots, \rho_{n}\right]
$$

and the sequence of the length $n$ for unitary transitions between observations

$$
\left[U_{1}, \ldots, U_{k}, \ldots, U_{n}\right] .
$$

The standard quantum mechanics presupposes a single unitary evolution, $U_{k}$, between observations at the times $t_{k-1}$ and $t_{k}$. The single-step transition probability takes the form

$$
\mathbf{P}_{k}=\operatorname{tr}\left(U_{k} \rho_{k-1} U_{k}^{\dagger} \rho_{k}\right) .
$$


Figure 1. Natural representation of $S_{N}$ decomposes into two irreducibles: 1D trivial and $(\mathrm{N}-1) \mathrm{D}$ standard representations.

Canonical bases:

in the trivial subspace

$e_{1}+e_{2}+\cdots+e_{\mathrm{N}}$

in the standard subspace

$e_{1}-e_{2}$

$e_{2}-e_{3}$

:

$e_{\mathrm{N}-1}-e_{\mathrm{N}}$

The evolution can be expressed via the Hamiltonian: $U_{k}=\mathrm{e}^{-\mathrm{i} H\left(t_{k}-t_{k-1}\right)}$. In physical theories, Hamiltonians are usually derived from the principle of least action, which, like any extremal principle, implies the selection of a small subset of dominant elements in a large set of candidates. Thus it is natural to assume that, in fact, all unitary evolutions take part in the transition between observations with their weights, but only the dominant evolutions are manifested in observations. Therefore, in our model, we use the following modification of the single-step transition probability

$$
\mathbf{P}_{k}=\sum_{m=1}^{\mathrm{M}} w_{k m} \operatorname{tr}\left(U_{k, m} \rho_{k-1} U_{k, m}^{\dagger} \rho_{k}\right),
$$

where $U_{k, m}=\mathrm{U}\left(g_{m}\right), g_{m} \in \mathrm{G} ; \mathrm{G}=\left\{g_{1}, \ldots, \mathrm{g}_{\mathrm{M}}\right\}$ is a finite group; $\mathrm{U}$ is a unitary representation of $\mathrm{G}$; $w_{k m}$ is the weight of the $m$ th group element at the $k$ th transition.

The operators $U_{k, m}$ that maximize $\operatorname{tr}\left(U_{k, m} \rho_{k-1} U_{k, m}^{\dagger} \rho_{k}\right)$ will be called dominant evolutions.

The single-step entropy is defined as $\Delta \mathbf{S}_{k}=-\log \mathbf{P}_{k}$.

The continuum approximation of (7) leads to a Lagrangian $\mathcal{L}$. Taking the logarithm of the probability of the whole trajectory, $\mathbf{P}_{0 \rightarrow n}=\prod_{k=1}^{n} \mathbf{P}_{k}$, we arrive at the entropy of the trajectory $\mathbf{S}_{0 \rightarrow n}=\sum_{k=1}^{n} \Delta \mathbf{S}_{k}$, the continuum approximation of which is the action $\mathcal{S}=\int \mathcal{L} d t$.

\subsection{Continuum approximation of a discrete model}

A continuum approximation of the above model requires the following simplifying assumptions:

- The sequence (2) should be replaced by a continuous time interval $\left[t_{0}, t_{n}\right] \subseteq \mathbb{R}$.

- The sequences (3) and (4) are to be replaced by continuous functions of time, $\rho(t)$ and $U(t)$.

- The relation $\operatorname{tr}\left(\rho^{2}\right)=1$ is necessary to ensure the continuity of the probability. This relation holds only for pure states $\rho=|\psi\rangle\langle\psi|$. So, we will consider $\psi$ instead of $\rho$.

- Assuming that $U$ belongs to a unitary representation of a Lie group, we use the Lie algebra approximation, $U \approx \mathbb{1}+\mathrm{i} A$, where $A=A(t)$ is a function the values of which are Hermitian matrices.

- We introduce derivatives and use the linear approximations $\Delta A \approx \dot{A} \Delta t$ and $\Delta \psi \approx \dot{\psi} \Delta t$. 
Applying these assumptions and approximations to the single-step entropy (7) and taking the infinitesimal limit we obtain the Lagrangian:

$$
\mathcal{L}=\underbrace{\left\langle\psi\left|\dot{A}^{2}\right| \psi\right\rangle-\langle\psi|\dot{A}| \psi\rangle^{2}}_{\text {dispersion of } \dot{A} \text { in the state } \psi}-\mathrm{i}(\langle\dot{\psi}|\dot{A}| \psi\rangle-\langle\psi|\dot{A}| \dot{\psi}\rangle+2\langle\psi|\dot{A}| \psi\rangle\langle\psi \mid \dot{\psi}\rangle)-\langle\psi \mid \dot{\psi}\rangle^{2} .
$$

\subsection{Dominant unitary evolutions in a symmetric group}

The dominant evolutions among the states represented by the vectors from the module $H=\mathbb{N}^{\mathrm{N}}$ for the group $S_{N}$ can be computed as follows.

$$
\text { Let }|n\rangle=\left(\begin{array}{c}
n_{1} \\
\vdots \\
n_{\mathrm{N}}
\end{array}\right),|m\rangle=\left(\begin{array}{c}
m_{1} \\
\vdots \\
m_{\mathrm{N}}
\end{array}\right),|1\rangle=\left(\begin{array}{c}
1 \\
\vdots \\
1
\end{array}\right) \text { be } \mathrm{N} \text {-dimensional vectors with natural components. }
$$

The Born probabilities for the pair $|n\rangle$ and $|m\rangle$ are

$$
\begin{aligned}
& \mathbf{P}_{\text {nat }}(|n\rangle,|m\rangle)=\frac{\langle n \mid m\rangle^{2}}{\langle n \mid n\rangle\langle m \mid m\rangle}-\text { natural representation, } \\
& \mathbf{P}_{\text {std }}(|n\rangle,|m\rangle)=\frac{\left(\langle n \mid m\rangle-\frac{1}{\mathrm{~N}}\langle n \mid 1\rangle\langle 1 \mid m\rangle\right)^{2}}{\left(\langle n \mid n\rangle-\frac{1}{\mathrm{~N}}\langle n \mid 1\rangle\langle 1 \mid n\rangle\right)\left(\langle m \mid m\rangle-\frac{1}{\mathrm{~N}}\langle m \mid 1\rangle\langle 1 \mid m\rangle\right)} \text { - standard representation. }
\end{aligned}
$$

Let $R_{a}$ denote the permutation (as well as its representation), that sorts the components of the vector $|a\rangle$ in some order. It is not hard to show that the unitary operator $U=R_{m}^{-1} R_{n}$ maximizes the probability $\mathbf{P}_{*}(U|n\rangle,|m\rangle)$, where the permutations $R_{n}$ and $R_{m}$ sort the vectors $|n\rangle$ and $|m\rangle$ identically in the case of a natural representation, and either identically or oppositely, depending on the value of the numerator in (8), in the case of a standard representation.

\subsection{Energy of permutation}

The Planck formula, $E=h v$, relates the energy to the frequency. This relation is reproduced by the quantum-mechanical definition of the energy as an eigenvalue of the Hamiltonian, $H=\mathrm{i} \hbar \ln U$, associated with a unitary transformation. Consider the energy spectrum of a unitary operator defined by a permutation. Let $p$ be a permutation of the cycle type $\left\{\boldsymbol{\ell}_{1}^{m_{1}}, \ldots, \boldsymbol{\ell}_{k}^{m_{k}}, \ldots, \boldsymbol{\ell}_{K}^{m_{K}}\right\}$, where $\boldsymbol{\ell}_{k}$ and $m_{k}$ represent lengths and multiplicities of cycles in the decomposition of $p$ into disjoint cycles. A short calculation shows that the Hamiltonian of the permutation $p$ has the following diagonal form

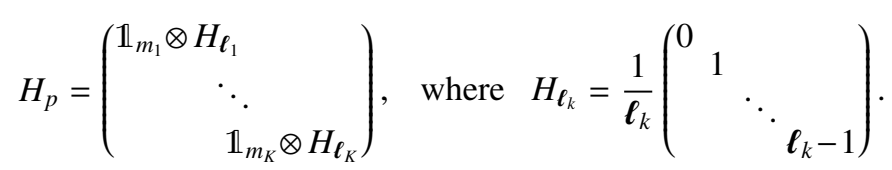

We shall call the least nonzero energy of a permutation the base energy:

$$
\boldsymbol{\varepsilon}=\frac{1}{\max \left(\boldsymbol{\ell}_{1}, \ldots, \boldsymbol{\ell}_{K}\right)} .
$$

The simulation shows that the base ("ground state", "zero-point", "vacuum") energy is statistically more significant than other energy levels.

\subsection{Monte Carlo simulation of dominant evolutions}

Figure 2 shows several dominant evolutions for the standard representation of the groups $S_{100}$ and $\mathrm{S}_{2000}$. Each graph represents the time dependencies of the Born probabilities for the dominant evolutions between four randomly generated pairs of natural vectors. The dominant evolutions are marked by labeling their peaks with their base energies: $\varepsilon \in\left\{\frac{1}{61}, \frac{1}{69}, \frac{1}{72}, \frac{1}{98}\right\}$ and $\varepsilon \in\left\{\frac{1}{1416}, \frac{1}{1789}, \frac{1}{1939}, \frac{1}{1972}\right\}$ for $S_{100}$ and $S_{2000}$, respectively. We see that with increasing the group size, non-dominant evolutions become almost invisible against the sharp peaks of the dominant evolutions. 

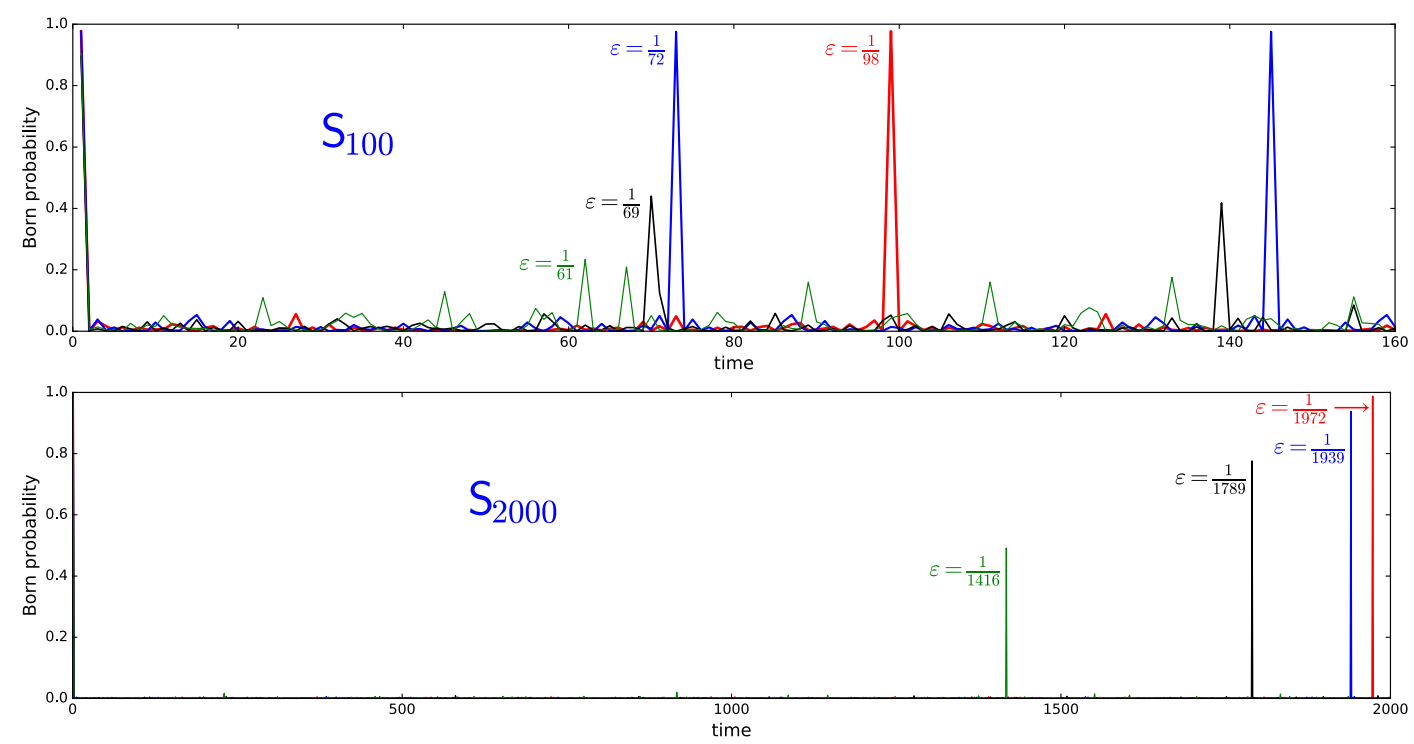

Figure 2. Dominant evolutions between randomly generated states. Born probability vs time

\section{Summary}

1. A constructive version of the quantum formalism can be formulated in terms of projections of permutations of finite sets onto invariant subspaces.

2. The quantum randomness is a consequence of the fundamental impossibility of tracing the individuality of indistinguishable entities in their evolution.

3. The natural number systems for the quantum formalism are the cyclotomic fields and the field of complex numbers is just their non-constructive metric completion.

4. The observable behavior of a quantum system is determined by the dominants among all possible quantum evolutions.

5. The principle of least action is a continuum approximation of the principle of selection of the most probable trajectories.

\section{References}

[1] A.M. Gleason, Indiana Univ. Math. J. 6, 885-893 (1957)

[2] M. Van Raamsdonk, Gen. Rel. Grav. 42, 2323-2329 (2010)

[3] J. Maldacena and L. Susskind, Fortschr. Phys. 61 781-811 (2013)

[4] C. Cao, S.M. Carroll, and S. Michalakis, Phys. Rev. D 95, 024031 (2017)

[5] V.V. Kornyak, EPJ Web of Conferences 108, 01007 (2016)

[6] V.V. Kornyak, Mathematical Modelling and Geometry 3, No 1, 1-24 (2015)

[7] V.V. Kornyak, Phys. Part. Nucl. 44, No 1, 47-91 (2013)

[8] R. Solomon, Bull. Amer. Math. Soc. (N.S.) 38, No 3, 315-352 (2001)

[9] Charles W. Curtis, Irving Reiner, Representation Theory of Finite Groups and Associative Algebras (John Wiley \& Sons, 1962). Reedition by AMS Bookstore, 2006. 